\title{
RÉGIMEN ACADÉMICO DOCENTE DE LA UNIVERSIDAD DE COSTA RICA
}

\author{
TENURE TRACK AT THE UNIVERSIDAD DE COSTA RICA
}

\author{
Volumen 12, Número 1 \\ Enero-Abril
}

pp. 1-14

Este número se publicó el 28 de febrero de 2012

\author{
Libia Herrero Uribe
}

Revista indizada en $\underline{\text { REDALYC }}$

Revista distribuida en las bases de datos:

CATÁLOGO DE LATINDEX, IRESIE, CLASE, DIALNET, DOAJ, E-REVIST@S,

Revista registrada en los directorios:

ULRICH'S, REDIE, RINACE, OEI, MAESTROTECA, PREAL, HUASCARAN, CLASCO 


\title{
RÉGIMEN ACADÉMICO DOCENTE DE LA UNIVERSIDAD DE COSTA RICA \\ TENURE TRACK AT THE UNIVERSIDAD DE COSTA RICA
}

\section{Libia Herrero Uribe ${ }^{1}$}

\begin{abstract}
Resumen: El Régimen Académico Docente de la Universidad de Costa Rica (UCR) es el sistema que le permite al profesorado universitario hacer carrera profesional docente y avanzar hacia diversas categorías con base en sus méritos académicos y en su experiencia universitaria. Las publicaciones, obras artísticas, didácticas o profesionales calificadas son el rubro más importante para aumentar el puntaje en el tiempo. Sin embargo, en el caso del profesorado en la categoría "catedrático", se ha estudiado si variables como el tipo de nombramiento y la jornada laboral, distribuida entre tiempos de investigación y de docencia, influyen en la cantidad de las publicaciones realizadas. En ese sentido, la información utilizada en este trabajo fue tomada de los registros de la Comisión de Régimen Académico Docente, en el mes de marzo del 2011, en la Sección de Cargas Académicas del Centro de Evaluación Académica (CEA), Vicerrectoría de Docencia, durante el primer ciclo del 2011. Dicho estudio ha demostrado que, en efecto, variables como el tipo de nombramiento, la jornada que labora y el tiempo dedicado a las labores docentes sí influyen en la cantidad de publicaciones que puede realizar un profesor o profesora universitaria. Adicionalmente, la forma en que el reglamento de Régimen Académico valora y cuantifica los trabajos, según el número de autores por publicación, es de igual forma una variable muy importante, porque incide en el puntaje que puede obtener el profesorado. Por lo anterior, se sugiere la incorporación del Portafolio de Vida Docente para que todo el profesorado sea evaluado con un mismo criterio, ya que el cuerpo docente debe impartir la docencia.
\end{abstract}

Palabras clave: RÉGIMEN ACADÉMICO, PUBLICACIONES, TIPO DE JORNADA, NOMBRAMIENTO, UNIVERSIDAD DE COSTA RICA

Abstract: The tenure track at the UCR valorates scientific publications and art pieces to upgrade its professors in their academic career. However, there are certain contractual conditions that may determine the final score an academic can obtain. These conditions are the number of teaching hours per week; the type of contract and type of appointments and the criteria to assess professors' works. The information presented in this paper was taken from the records of the Vicepresidency of Academic Affairs at UCR. This work has determined that the type of contract, the amount of teaching hours per week and the way publications are assessed are important factors that have a direct impact in a Professor academic career and upgrades. Since teaching is a compulsory activity for academics at this university, but research has a heavier weight in Professors assessment, the author suggests the incorporation of the Academic Life Portfolio (Portafolio de Vida Docente) as a criteria for advancing in academic career so that teaching and pedagogy become as important mechanism as research in academic track and upgrades.

Key Words: TENURE TRACK, ACADEMIC CAREER, PUBLICATIONS, LABOR CONTRACTS, APPOINTMENTS, UNIVERSITY OF COSTA RICA

\footnotetext{
1 Doctora en Virología, Universidad de Londres, Inglaterra. Docente de la Cátedra de Virología Médica, Investigadora del Centro de Investigaciones en Enfermedades Tropicales, Facultad de Microbiología. Actualmente Vicerrectora de Docencia de la Universidad de Costa Rica.
}

Dirección electrónica: libia.herrero@ucr.ac.cr

Artículo recibido: 16 de setiembre, 2011

Aprobado: 26 de enero, 2012 


\section{Introducción}

El Régimen Académico (RA) Docente de la Universidad de Costa Rica (UCR) es el sistema que le permite al profesorado universitario hacer carrera profesional docente y avanzar a diversas categorías con base en sus méritos académicos y en su experiencia universitaria. La Comisión de Régimen Académico, nombrada por el Consejo Universitario, es la encargada de valorar los antecedentes del profesorado que ha ingresado al régimen para establecer la categoría que le corresponde. Dicha Comisión realiza su función de acuerdo con las disposiciones de un Reglamento específico para tal efecto y es la máxima autoridad en este campo. Sus decisiones sólo podrán ser apeladas ante el Consejo Universitario.

Las categorías de la carrera profesional docente del profesorado universitario son cuatro: Instructor, Adjunto, Asociado y Catedrático. El ingreso a Régimen Académico se logra como resultado de la participación en un concurso de antecedentes. La primera categoría que se adquiere es la de Instructor, ésta se mantiene hasta tanto se solicite a la Comisión de Régimen el ascenso para pasar a la que corresponda. Todo de acuerdo con los procedimientos del Reglamento.

La Comisión para otorgar un ascenso en el régimen toma en cuenta lo siguiente: a) la condición académica, que incluye los grados académicos, a los cuales se les asigna un puntaje según su complejidad, b) el tiempo de servicio en la docencia universitaria, c) la evaluación del profesor en la docencia, la investigación y la acción social, d) la experiencia universitaria: publicaciones, obras artísticas, didácticas o profesionales calificadas y e) el conocimiento de idiomas. Todos los rubros tienen un puntaje límite, excepto los puntos $\mathrm{d}$ y e citados anteriormente, que pueden seguir aumentando con el tiempo.

Un rubro muy importante para lograr el ascenso en Régimen son las publicaciones en revistas o libros indexados en listas internacionales; así como obras profesionales, artísticas y didácticas. Según el reglamento de esta instancia se otorga un puntaje de 0 a 4 puntos a cada obra o publicación dependiendo de la calidad de ésta. En el caso de obras y publicaciones de varios autores, el puntaje se distribuye de acuerdo con el grado de participación de los autores. Cuando el trabajo es de varias personas, se otorga al solicitante, en forma adicional, un $25 \%$ del puntaje que le corresponde por su grado de participación, siempre que la suma de ambos no exceda el puntaje total asignado a la obra o publicación. Las publicaciones y las obras de uno o varios autores pueden recibir 
calificaciones en números enteros o fracciones: 0 puntos, si se considera que el trabajo tiene poco impacto, o si la participación de los coautores está muy diluida. Hasta 1 punto, cuando se considere que el trabajo tiene relevancia en su campo, hasta 2 puntos, publicaciones y obras de mérito reconocido y hasta 4 puntos, a publicaciones y obras que por su contenido se consideren de importante y excepcional trascendencia en su campo. Este puntaje se podrá aumentar a criterio de la Comisión, en números enteros hasta 6 puntos por obra 0 publicación de gran complejidad, originalidad, trascendencia y reconocido mérito.

El solicitante para tener derecho al ascenso por este rubro específicamente deberá satisfacer el siguiente valor numérico mínimo: para ascender a Profesor Adjunto se necesitan 4 puntos, para Profesor Asociado 8 puntos y para Profesor Catedrático 16 puntos. Sin embargo, para tener derecho al ascenso de una categoría a otra, el valor numérico total que se necesita es el siguiente: de Instructor a Profesor Ajunto se necesitan 36 puntos, de Profesor Adjunto a Profesor Asociado 54 puntos, de Profesor Asociado a Catedrático 90 puntos.

El rubro más importante para aumentar el puntaje es la experiencia universitaria, es decir, publicaciones, obras artísticas, didácticas o profesionales calificadas. Sin embargo, existen varios factores que pueden incidir en la dedicación del profesorado a estas actividades, como por ejemplo, el tipo de nombramiento: parcial o completo, si es propietario o interino y la carga docente en su plan de trabajo, entre otras.

Otro factor que puede incidir en el puntaje del profesorado es la forma en que se valoran los trabajos publicados, siendo los libros y la publicación de un solo autor los que obtienen los puntajes más altos y los trabajos multidisciplinarios son "castigados" según el número de personas en la autoría de la publicación.

El objetivo de este trabajo es presentar el número de profesores y profesoras catedráticos de la Universidad de Costa Rica de acuerdo con el puntaje en Régimen Académico, por área de conocimiento, por la jornada de trabajo y por el sexo, esto con el fin de analizar si estos factores afectan el puntaje total y si la forma de evaluar las publicaciones, obras artísticas, didácticas o profesionales calificadas, influye en los puntajes adquiridos por el profesorado de las diferentes áreas del conocimiento. 


\section{Fuentes y Metodología}

La información utilizada en este trabajo fue tomada de los registros de la Comisión de Régimen Académico Docente, en el mes de marzo del 2011 y de la Sección de Cargas Académicas del Centro de Evaluación Académica (CEA), Vicerrectoría de Docencia, durante el primer ciclo del 2011.

Es importante aclarar que los datos de Régimen Académico varían constantemente, ya que el profesorado solicita evaluación de sus atestados constantemente, y los datos de la Sección de Cargas Académicas varían semestre a semestre. A partir de estas fuentes se calcularon los datos que se presentan en este trabajo. En la UCR se tienen dos tipos de nombramientos: el propietario, cuando el docente ocupa la titularidad de una plaza o fracción de ésta en una determinada categoría de Régimen Académico y el interino, donde el docente ocupa una plaza o fracción de ésta, con o sin la adquisición de la condición de relación laboral continua, pero sin la posibilidad de pertenecer a Régimen Académico.

Cuando se menciona docencia, se refiere a la docencia directa e indirecta que realiza el o la profesora en su plan de trabajo semanal.

\section{Resultados}

El tipo de nombramiento del profesorado puede ser un factor importante en la producción científica, artística o profesional. En la Figura No.1, se observa que el $21.8 \%$ del total del profesorado tiene un nombramiento de tiempo completo, siendo la jornada de un cuarto de tiempo el nombramiento más frecuente en nuestra Institución, y solo el 33\% del profesorado tiene nombramiento en propiedad.

El profesorado en Régimen Académico en la UCR durante el primer ciclo del año 2011 fue de 1681 profesores y profesoras, de los cuales el 25.9\% pertenecieron al área de Ciencias Sociales (Cuadro No.1), el $24.1 \%$ del área de la Salud y el $14.8 \%$ de Artes y Letras. Sin embargo, si se cuantifica el profesorado en la categoría de catedrático, con 90 o más puntos, es el área de Ciencias Básicas la que tiene el porcentaje más alto de profesores en esta categoría (51.9\%). Luego, le sigue Artes y Letras con un $38.1 \%$ y con un $37.2 \%$, Agroalimentarias. En el mismo cuadro se observa el porcentaje del profesorado con $100 \mathrm{o}$ más puntos en Régimen Académico, teniendo Ciencias Básicas el porcentaje más alto (31.5\%), seguido por Artes y Letras con un $27.3 \%$ y Ciencias Sociales de tercera con un $21.3 \%$ de su profesorado. 
Los diez puntajes más altos obtenidos por el profesorado en las diferentes áreas se muestran en el Cuadro No.2, donde se observa que son las profesoras y profesores del área de Artes y Letras quienes tienen los puntajes más altos, seguido por Ciencias Sociales y Ciencias Básicas. El porcentaje de los grados académicos del profesorado con los puntajes más altos por área se muestra en el Cuadro No.3, donde se observa que en el área de Artes y Letras solo el $40 \%$ de sus catedráticos tienen el grado de doctor, porcentaje igual al profesorado con el grado de Licenciatura, mientras que en el resto de las áreas, el $90 \%$ del profesorado tiene posgrado.

La jornada de trabajo del profesorado es un factor muy importante, pues entre más dedicación tenga a la academia, más posibilidades tiene de investigar y publicar sus trabajos. En la UCR existe gran variación en la jornada de trabajo en las diferentes Unidades Académicas como se observa en el Cuadro No.4; las escuelas de Química, Agronomía y Filología y Lingüística se encuentran en los tres primeros lugares con respecto a profesores con nombramientos de tiempo completo comparado con Administración de Negocios y Medicina que solo el $4 \%$ de sus profesores tienen ese tipo de nombramiento.

Otro factor muy importante para tomar en cuenta en la producción científica, artística y profesional es el porcentaje del profesorado con nombramiento en propiedad o interino. La escuela de Agronomía es la unidad académica que tiene el porcentaje más alto de docentes en propiedad, seguida de la escuela de Biología y Estudios Generales. Por su parte, las escuelas con los menores porcentajes de docentes en propiedad son Formación Docente, las Sedes Regionales y Farmacia (Cuadro No.5).

Para lograr relacionar algunas de las variables estudiadas en este trabajo, se identificaron las escuelas de donde provenían los y las profesoras con los puntajes más altos en Régimen Académico.

En el área de la Salud se encontraron 6 docentes de la Facultad de Microbiología y 3 de la Escuela de Medicina, mientras que en el área de Artes y Letras los dos primeros lugares estuvieron en las escuelas de Estudios Generales y Filología y Lingüística con 3 cada una. En el área de Ciencias Básicas, la escuela de Biología contó con 3 docentes, similar al área de las Ingenierías, donde la escuela de Ciencias de la Computación e Informática contó con 3 docentes y el resto estuvo repartido entre las otras escuelas de las dos áreas. En el caso del área de las Agroalimentarias, 7 de los profesores y las profesoras 
con los mejores puntajes fueron de la escuela de Agronomía y en el área de Ciencias Sociales 4 docentes fueron de la Facultad de Derecho.

En las Sedes Regionales, 5 del profesorado fueron de la Sede de Occidente, 2 del Pacífico y 2 del Atlántico. Se determinó también que el $94.6 \%$ de este grupo cuentan con jornadas de tiempo completo. Esta información no se muestra en el trabajo.

La carga en docencia directa e indirecta en que esté involucrado el profesor o la profesora incide en la producción científica, artística y profesional del profesorado. El Cuadro No.6 muestra el porcentaje de horas dedicadas a la docencia directa e indirecta reportadas por las Unidades Académicas en el Plan de Trabajo semanal de su profesorado.

Las escuelas de Estudios Generales, Medicina y Administración de Negocios ocupan alrededor del 90\% de sus tiempos docentes en docencia directa o indirecta, mientras que las escuelas de Biología y Agronomía ocupan el 37.4\% y 30.2\% respectivamente en este rubro, teniendo más tiempo el profesorado para dedicarse a la investigación, acción social y por lo tanto, a publicar. El área de las Ciencias Básicas es la que tiene asignada menos carga o horas para la docencia directa e indirecta en su plan de estudios, con excepción de la escuela de Matemáticas, ya que su profesorado tiene una carga docente del 64\%, muy cercano al promedio de la Institución.

Al analizar el índice de masculinidad entre el grupo de catedráticos y catedráticas por área de conocimiento, se observa un predominio de los varones en todas las áreas, con excepción del área de la Salud (Cuadro No.7).

\section{Discusión}

La Universidad de Costa Rica es una institución que conjuga tres actividades fundamentales con el fin de lograr una educación pertinente, integral y de calidad: la docencia, la investigación y la acción social. Como institución de Educación Superior, tiene un cuerpo de académicos dedicados a las tres actividades fundamentales en las cuales son evaluados, además, el desarrollo de méritos como el grado académico, el conocimiento de idiomas y la pertinencia y calidad de sus publicaciones.

El rubro más importante para aumentar el puntaje en Régimen Académico en el tiempo es la experiencia universitaria, la cual se refleja en publicaciones, obras artísticas, didácticas o profesionales calificadas. Sin embargo, existen varios factores que inciden en la 
producción científica como el tipo de nombramiento, la jornada y la carga en docencia directa e indirecta.

El análisis de las características del profesorado que se encuentra en los diez primeros lugares en las diferentes áreas del conocimiento de la Institución han demostrado que tener un nombramiento de tiempo completo $(94.6 \%)$ es muy importante para poder dedicarse a escribir y publicar sus experiencias. Sin embargo, en el área de Ciencias Sociales, específicamente, 3 profesores de la Facultad de Derecho tienen nombramientos parciales.

El grado académico del profesorado con los puntajes más altos es en su mayoría el de doctorado, como se muestra en el cuadro 3, sin embargo, en el área de Artes y Letras hay 4 profesores con licenciatura, lo que demuestra que si se publican suficientes trabajos y, además, se hace bajo un solo autor, es posible llegar a tener un puntaje alto en Régimen Académico con el grado de licenciatura.

La Escuela de Agronomía agrupa tres variables muy importantes: alto porcentaje del profesorado con nombramientos de tiempo completo y en propiedad y, de igual modo, una carga en docencia directa e indirecta baja, características óptimas para investigar, hacer acción social y, por consiguiente, escribir y publicar, traduciéndose esto en puntajes más altos, lo que 7 de 10 profesores(as) de Agronomía logra; sin embargo, los puntajes del profesorado del área de Ciencias Agroalimentarias no son tan altos como en el resto de las áreas (Cuadro No.2). Esto podría ser debido a que tengan menos publicaciones o que éstas sean realizadas por un grupo de investigadores(as), en cuyo caso se recibe un menor puntaje.

El grupo del profesorado que se encuentra con más desventaja para poder publicar son aquellos que están nombrados parcialmente, o profesores de tiempo completo, en propiedad o interinos, con cargas muy altas en docencia directa o indirecta, como en las escuelas de Medicina y Administración de Negocios (Cuadro No.6) que además de la alta carga en docencia, tienen el porcentaje menor de su profesorado de tiempo completo. No obstante, es importante hacer notar que los tres profesores de la escuela de Medicina con los puntajes más altos en el área de la Salud tienen jornadas de tiempo completo y nombramientos en propiedad.

Las escuelas que dedican porcentajes altos de su presupuesto en docencia directa o indirecta, pero con un porcentaje alto del profesorado de tiempo completo, permiten un ambiente más universitario de intercambio de ideas y colaboración que al final se traduce en 
un número mayor de publicaciones y una carga distributiva entre los profesores que les permite otras actividades además de la docencia como lo demuestra la escuela de Estudios Generales, que aunque ocupe el 90\% de su carga en docencia directa e indirecta, cuatro de sus profesores(as) cuentan con los puntajes más altos del área de Artes y Letras. Sin embargo, estos profesores podrían ser aquellos que publican con más frecuencia como autor único, logrando puntajes más altos en Régimen Académico.

Merece un comentario especial el índice de masculinidad en los y las catedráticas por área del conocimiento, ya que con excepción del área de la Salud, en el resto de las áreas predominan los varones. Si se compara con los datos obtenidos por Garita y Herrero en el año 2005, se logra un aumento significativo de mujeres catedráticas en el área de la Salud, le siguen las áreas de Ingeniería y Arquitectura y las Ciencias Básicas y en el resto de las áreas se observa un aumento leve en el número de varones con respecto al año 2005 . No obstante, para el año 2010, las áreas con índice de masculinidad más alto son Ciencias Básicas y Ciencias Agroalimentarias. Este cuadro demuestra los cambios tan importantes en este índice, que aunque cada vez más tiende a que se logre un balance entre los géneros, es evidente que las mujeres tienen menos tiempo para investigar y escribir, algunas razones pueden ser que, en general, en las comisiones de las escuelas y facultades hay un predominio de participación de las mujeres y, desde luego, por sus labores domésticas y de cuido pueden repercutir en sus labores universitarias.

Este trabajo ha demostrado que variables como el tipo de nombramiento, jornada que labora y el tiempo dedicado a las labores docentes influyen de manera significativa en la cantidad de publicaciones que puede realizar un profesor o profesora universitaria. Adicionalmente, la forma en que el reglamento de Régimen Académico valora y cuantifica los trabajos según el número de autores por publicación es una variable muy importante que incide en el puntaje que puede obtener el profesorado en Régimen Académico. Sin embargo, también se demuestra que los individuos hacen la diferencia, ya que aunque no tengan el grado académico de Doctor o laboren un tiempo completo también logran estar entre los puntajes más altos.

Lo relevante es que existe un reglamento de Régimen Académico Docente único que sirve para categorizar a los profesores que no se encuentran en igualdad de condiciones, pues el número de tiempos completos, nombramientos totales o parciales y la carga asignada a cada profesor o profesora en docencia directa o indirecta depende de aspectos 
históricos, disciplinares y presupuestales. Es evidente, también, que el reglamento favorece a las publicaciones de un solo autor o autora; que al artista se le debe honrar su creatividad individual, y que debe estimular el trabajo y la investigación de equipos inter, multi y transdiciplinares.

La normativa universitaria establece que todo profesor o profesora debe hacer docencia; por lo tanto, la UCR debería considerar darle más puntaje a los procesos de enseñanza-aprendizaje y las innovaciones en el aula, y no solo evaluar la actividad docente. Una sugerencia interesante de valorar es la incorporación del Portafolio de Vida Docente, un medio de autoevaluación y autogestión, que puede ser evaluado por pares y darle un valor al proceso de reflexión del docente y a la incorporación de innovaciones. De esta forma, el Régimen Académico Docente sería más justo, valoraría la actividad sustantiva de todo el profesorado y daría la oportunidad de incrementar la creatividad e innovación dentro y fuera del aula.

\section{Referencias}

Costa Rica, Universidad de Costa Rica. (1985). Reglamento de Régimen Académico y Servicios Docente. San José, C.R: publicado en la Gaceta Universitaria 16-85, con fecha 19-12-85.

Garita Bonilla, Nora y Herrero Uribe, Libia. (2005). Mujer y Ciencia en la Universidad de Costa Rica. Revista Centroamericana de Ciencias Sociales, II(2), 79-101. 


\section{Cuadro No.1 \\ Porcentajes de profesores en Régimen Académico por Área y catedráticos con puntajes $\geq 90$ y $\geq 100$ puntos \\ Marzo, 2011}

\begin{tabular}{|l|c|c|c|c|}
\hline \multicolumn{1}{|c|}{ Área Académica } & \multicolumn{2}{|c|}{$\begin{array}{c}\text { Profesores en } \\
\text { Régimen } \\
\text { Académico } \\
\text { ABS. }\end{array}$} & $\begin{array}{c}\text { REL. } \\
\text { Catedráticos con } \\
\text { Puntaje } \mathbf{2 9 0}\end{array}$ & $\begin{array}{c}\% \text { Catedráticos con } \\
\text { Puntaje } \geq \mathbf{1 0 0}\end{array}$ \\
\hline Artes y Letras & 249 & 14,8 & $38,1 \%$ & $27,3 \%$ \\
\hline Ciencias Básicas & 152 & 9,0 & $51,9 \%$ & $31,5 \%$ \\
\hline Ciencias Sociales & 436 & 25,9 & $32,5 \%$ & $21,3 \%$ \\
\hline Agroalimentaria & 94 & 5,5 & $37,2 \%$ & $10,6 \%$ \\
\hline Ingenierías y Arq & 174 & 10,3 & $21,2 \%$ & $7,4 \%$ \\
\hline Salud & 406 & 24,1 & $16,7 \%$ & $10,0 \%$ \\
\hline Sedes Regionales & 170 & 10,1 & $13,5 \%$ & $100 \%$ \\
\hline TOTAL UCR & 1681 & 100,00 & $100 \%$ & \\
\hline
\end{tabular}

Fuente: Sección Régimen Académico (CEA). 


\section{Catedráticos con los 10 puntajes más altos en Régimen Académico por Área}

Marzo, 2011

\begin{tabular}{|c|c|c|c|c|c|c|c|}
\hline Área & $\begin{array}{l}\text { Artes y } \\
\text { Letras }\end{array}$ & $\begin{array}{l}\text { Ciencias } \\
\text { Sociales }\end{array}$ & $\begin{array}{l}\text { Ciencias } \\
\text { Básicas }\end{array}$ & Salud & $\begin{array}{c}\text { Sedes } \\
\text { Regionales } \\
\end{array}$ & $\begin{array}{l}\text { Ingeniería y } \\
\text { Arquitectura }\end{array}$ & $\begin{array}{c}\text { Ciencias } \\
\text { Agroalimentarias } \\
\end{array}$ \\
\hline $\begin{array}{c}\text { Los } 10 \\
\text { puntajes más } \\
\text { altos }\end{array}$ & $\begin{array}{l}263.25 \\
238,75 \\
207,00 \\
200,00 \\
185,25 \\
178,00 \\
161,40 \\
157,00 \\
156,00 \\
153,50\end{array}$ & $\begin{array}{l}243,00 \\
233,50 \\
200,00 \\
196,94 \\
190,50 \\
188,50 \\
177,75 \\
176,27 \\
175,50 \\
173,50\end{array}$ & $\begin{array}{l}219,25 \\
212,32 \\
204,00 \\
172,41 \\
168,57 \\
167,50 \\
164,00 \\
163,25 \\
163,19 \\
151,07\end{array}$ & $\begin{array}{l}172,00 \\
158,50 \\
153,79 \\
148,00 \\
135,30 \\
119,13 \\
118,74 \\
116,28 \\
111,89 \\
111,00\end{array}$ & $\begin{array}{l}169,42 \\
163,50 \\
157,94 \\
154,00 \\
149,00 \\
138,63 \\
136,25 \\
123,00 \\
119,50 \\
114,13\end{array}$ & $\begin{array}{l}163,49 \\
135,50 \\
133,25 \\
131,75 \\
126,50 \\
126,00 \\
122,00 \\
121,00 \\
120,00 \\
119,00\end{array}$ & $\begin{array}{l}153,50 \\
117,00 \\
117,00 \\
113,00 \\
112,00 \\
111,33 \\
108,00 \\
106,08 \\
106,00 \\
104,38\end{array}$ \\
\hline
\end{tabular}

Fuente: Sección Régimen Académico (CEA). 


\section{Cuadro No.3 \\ Grados Académicos de los Catedráticos con los diez puntajes más altos por Área (\%) Marzo, 2011}

\begin{tabular}{|c|c|c|c|}
\hline Área & Licenciatura & Magister & Doctorado \\
\hline Artes y Letras & 40 & 20 & 40 \\
\hline Ciencias Sociales & - & 10 & 90 \\
\hline Ciencias Básicas & - & 40 & 60 \\
\hline Ciencias Agroalimentarias & 10 & 20 & 70 \\
\hline Ingenierías y Arquitectura & - & 20 & 80 \\
\hline Salud & 10 & 10 & 80 \\
\hline Sedes Regionales & 10 & 10 & 80 \\
\hline Total & 10 & 18.6 & 71.4 \\
\hline
\end{tabular}

Fuente: Sección Técnica de Cargas Académicas (CEA).

\section{Cuadro No.4}

Porcentaje del total del profesorado con una jornada de Tiempo Completo, por Área y por Unidad Académica

I Ciclo 2011

\begin{tabular}{|c|c|c|c|c|c|}
\hline 1. Por Áreas & \multicolumn{3}{|c|}{ b) $>40 \%$} & \multicolumn{2}{|l|}{ c) $<10 \%$} \\
\hline Área & $\%$ & Unidad Académica & $\%$ & Unidad Académica & $\%$ \\
\hline Agroalimentarias & 48.2 & Química & 71.7 & Administración Pública & 9.5 \\
\hline Ciencias Básicas & 47.1 & Agronomía & 61.4 & Tecnologías en Salud & 9.1 \\
\hline & & Filología y Lingüística & 59.6 & Derecho & 8.4 \\
\hline Artes y Letras & 39.3 & Enfermería & 57.4 & Ingeniería Topográfica & 5.4 \\
\hline $\begin{array}{l}\text { Ingenierías } \\
\text { Arquitectura }\end{array}$ & 24.7 & Computación e Informática & 52.9 & Salud Pública & 5.1 \\
\hline Ciencias Sociales & 23.9 & Estudios Generales & 51.6 & $\begin{array}{l}\text { Administración } \\
\text { Negocios }\end{array}$ & 4.4 \\
\hline Sedes & 16.4 & Educación Física y Deportes & 51.4 & Medicina & 4.3 \\
\hline Salud & 13.6 & Artes Plásticas & 44.2 & & \\
\hline $\begin{array}{l}\text { Universidad de Costa } \\
\text { Rica }\end{array}$ & 21.8 & Microbiología & 40.8 & & \\
\hline
\end{tabular}

Fuente: Sección Técnica de Cargas Académicas (CEA). 
Cuadro No.5

Porcentaje del profesorado en propiedad, por Área y por Unidad Académica

I Ciclo 2011

a) Por Áreas

b) $\geq 45 \%$

c) $<20 \%$

\begin{tabular}{|c|c|c|c|c|c|}
\hline Área & $\%$ & $\begin{array}{l}\text { Unidad } \\
\text { Académica }\end{array}$ & $\%$ & Unidad Académica & $\%$ \\
\hline Agroalimentarias & 44.6 & Agronomía & 67.4 & Trabajo Social & 19.6 \\
\hline Artes y Letras & 36.9 & Biología & 64.5 & Orientación y Educación Especial & 17.8 \\
\hline Ciencias Básicas & 36.1 & $\begin{array}{l}\text { Estudios } \\
\text { Generales }\end{array}$ & 56.2 & Farmacia & 14.7 \\
\hline Ciencias Sociales & 30.8 & $\begin{array}{ll}\text { Filología } & \text { y } \\
\text { Lingüística } & \end{array}$ & 48.0 & Sedes Regionales & 13.7 \\
\hline Ingenierías & 29.1 & Historia & 46.1 & Formación Docente & 12.5 \\
\hline Salud & 24.5 & & & & \\
\hline Sedes Regionales & 13.7 & & & & \\
\hline $\begin{array}{c}\text { Universidad de } \\
\text { Costa Rica }\end{array}$ & 23.7 & & & & \\
\hline
\end{tabular}

Fuente: Sección Técnica de Cargas Académicas (CEA).

Cuadro No.6

Docencia (directa e indirecta) en el plan de trabajo semanal del profesorado por Escuela (\%) I Ciclo 2011

\begin{tabular}{|l|c|}
\hline \multicolumn{1}{|c|}{ Unidad Académica } & $\%$ \\
\hline Medicina & 91.7 \\
\hline Estudios Generales & 90.2 \\
\hline Administración de Negocios & 89.2 \\
\hline Ingeniería Topográfica & 84.4 \\
\hline Administración Pública & 82.6 \\
\hline Orientación y Educación Especial & 72.6 \\
\hline Computación e Informática & 67.4 \\
\hline Educación Física y Deportes & 66.9 \\
\hline Derecho & 66.4 \\
\hline Filología y Lingüística & 66.3 \\
\hline Historia & 63.2 \\
\hline Artes Dramáticas & 61.1 \\
\hline Formación Docente & 60.8 \\
\hline Trabajo Social & 57.4 \\
\hline Facultad de Microbiología & 48.4 \\
\hline Química & 46.4 \\
\hline Facultad de Farmacia & 44.2 \\
\hline Biología & 37.4 \\
\hline Agronomía & 30.2 \\
\hline Universidad de Costa Rica & 66.3 \\
\hline
\end{tabular}

Fuente: Sección Técnica de Cargas Académicas (CEA). 


\section{Cuadro No.7 \\ Índice de Masculinidad en el profesorado con puntaje $\geq 90$ por Área Académica 2010}

\begin{tabular}{|l|c|c|c|}
\hline \multicolumn{1}{|c|}{ Área } & $\mathbf{1 9 8 8}^{*}$ & $\mathbf{2 0 0 5}^{\boldsymbol{*}}$ & $\mathbf{2 0 1 0}$ \\
\hline Ciencias Sociales & 3.4 & 1.68 & 1.8 \\
\hline Artes y Letras & 2.2 & 1.5 & 1.6 \\
\hline Ciencias Básicas & 8.5 & 6.7 & 5.5 \\
\hline Salud & 11.1 & 2.1 & 0.86 \\
\hline Ingenierías y Arquitectura & 22 & 5.5 & 3.6 \\
\hline Ciencias Agroalimentarias & 19 & 3.6 & 4.6 \\
\hline Sedes Regionales & 6 & 2.5 & 2.6 \\
\hline
\end{tabular}

Fuente: *Garita y Herrero, 2005

Figura No.1

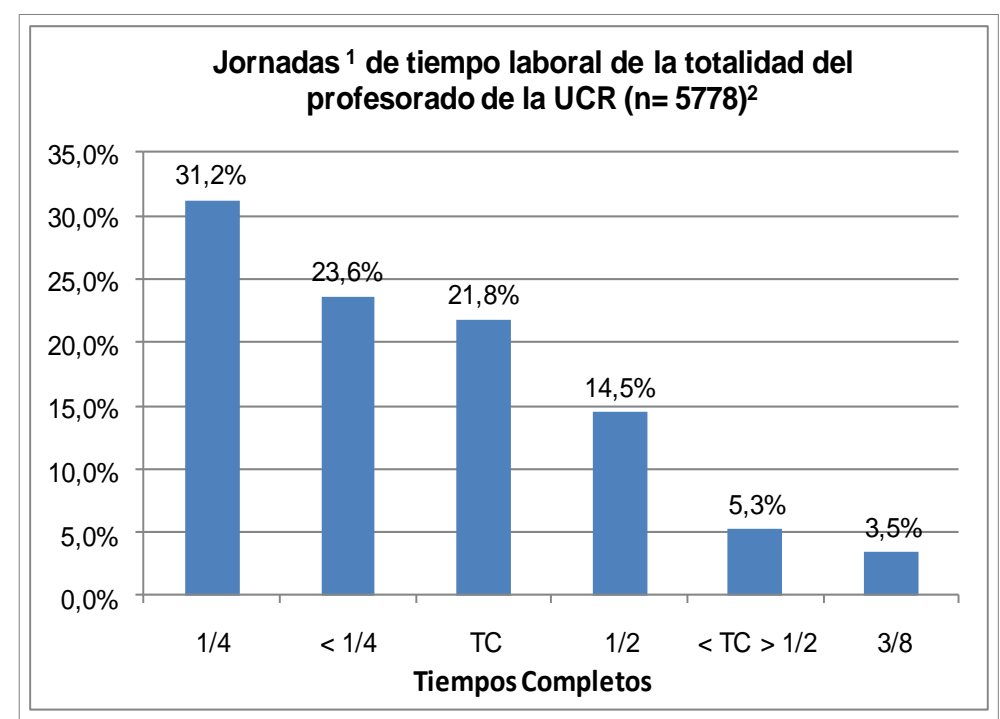

${ }^{1}$ Los docentes pueden tener uno o más nombramientos o jornadas.

2 Corresponde a la sumatoria de los datos de todas las unidades docentes y de investigación. Contabiliza las diferentes jornadas que tiene un docente en diferentes Unidades Académicas para completar su T.C. 